\title{
Parámetros antropométricos de atletas paralímpicos usuarios de silla de ruedas de la región del Biobío
}

\section{Anthropometric parameters of Paralympic athletes wheelchair users of the Biobío region}

\author{
*Yazmina Virginia Pleticosic Ramírez, *Cristián Luarte Rocha, \\ **Luis Felipe Castelli Correia De Campos, *Cinthya Barra Danyau, \\ *Valentina Pavez Van Rysselberghe
}

Pleticosic, Y., Luarte, C., Castelli, L., Barra, C., \& Pavez, V. (2019). Parámetros antropométricos de atletas paralímpicos usuarios de silla de ruedas de la región del Biobío. Revista Ciencias de la Actividad Física UCM, N²0(2), julio-diciembre, 1-14. DOI: http://doi.org/10.29035/rcaf.20.2.4

\section{RESUMEN}

El presente estudio, tuvo como objetivo determinar el índice de masa corporal (IMC) y porcentaje de grasa corporal (\%GC) de los atletas con proyección paralímpica, usuarios de sillas de ruedas, pertenecientes a la región del Biobío. El estudio fue de diseño no experimental-transversal de alcance descriptivo. La evaluación antropométrica se realizó a través del protocolo ISAK. Se determinó peso y estatura utilizando una balanza con tallímetro (Seca 220), circunferencia de cintura con una cinta Lufkin y 6 pliegues con un plicómetro Harpenden (0,2 mm). El porcentaje de grasa corporal se determinó, por medio de las ecuaciones de Durnin and Womersley y Siri. Los atletas en silla de ruedas pre-

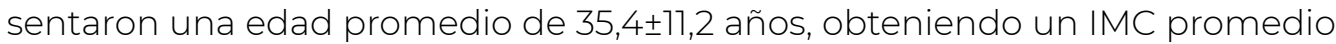

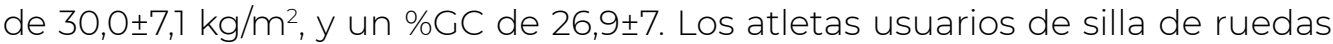
con proyección paralímpica, presentan un alto porcentaje de grasa corporal y clasificaciones de IMC y \%GC que los categorizan en personas que presentan obesidad.

\section{Palabras clave}

Antropometría, atletas, silla de ruedas.

\footnotetext{
* Universidad San Sebastián. Concepción, Chile.

** Universidad del Biobío. Chillán, Chile.
} 
Pleticosic, Y., Luarte, C., Castelli, L., Barra, C., \& Pavez, V. (2019). Parámetros antropométricos de atletas paralímpicos usuarios de silla de ruedas de la región del Biobío. Revista Ciencias de la Actividad Física UCM, N²0(2), julio-diciembre, 1-14.

DOI: http://doi.org/10.29035/rcaf.20.2.4

\section{ABSTRACT}

The objective of this study was to determine the body mass index (BMI) and body fat percentage of athletes with Paralympic projection, wheelchair users, belonging to the Biobío region. The study was of a non-experimental crosssectional design with a descriptive scope. The anthropometric evaluation was carried out through the ISAK protocol. Weight and height were determined using a balance with stadiometer (Seca 220), waist circumference with a Lufkin tape and 6 folds with a Harpenden pichometer $(0.2 \mathrm{~mm})$. The percentage of body fat was determined, by means of the Durnin and Womersley equations and Siri. The athletes in wheelchairs presented an average age of $35.4 \pm$ 11.2 years, obtaining an average BMI of $30.0 \pm 7.1 \mathrm{~kg} / \mathrm{m} 2$, and a GC\% of $26.9 \pm$ 7. Athletic wheelchair users with Paralympic projection, have a high body fat percentage and $\mathrm{BMI}$ ratings and $\mathrm{GC} \%$ that categorize them as people who are obese.

\section{Key words}

Anthropometry, athletes, wheelchair.

\section{INTRODUCCIÓN}

El deporte adaptado, según Hernández (2000), despliega una serie de funciones, por ejemplo, sensibilizar a la sociedad sobre los problemas de las personas con discapacidad y especialmente sobre la práctica deportiva. De forma peculiar, uno de los principales factores relacionados al deporte adaptado y paralímpico es que la persona en situación de discapacidad puede reconstruir su identidad, transparentar sus potencialidades y reducir significativamente sus limitaciones que, en su mayoría, es establecida por una interacción ne- gativa entre la persona y su contexto. Torralba, Braz y Rubio (2014) consideran que el deporte adaptado entrega los mismos beneficios en los aspectos físicos, psicológicos y sociales para las personas con discapacidad, que aquellos deportes convencionales que realizan las personas sin discapacidad.

El término deporte adaptado aborda todas las modalidades deportivas que se adaptan al colectivo de la persona en situación de discapacidad o condición especial, bien porque se 
han realizado una serie de adaptaciones $y / o$ modificaciones para facilitar la práctica de ese colectivo, o porque la propia estructura del deporte permite su práctica sin adaptaciones (Hernández, 2000; Pérez, Reina y Sanz, 2012; Reina, 2010). De esta forma, los deportes convencionales han adaptado una serie de parámetros para ajustarse a las necesidades del colectivo o disciplina deportiva que lo va a practicar. Estas modificaciones se orientan, de acuerdo a lo señalado por Hernández (2000), a sus reglas materiales, instalaciones, número de deportistas y apoyos, entre otros, que lo diferencian del practicado por personas sin discapacidad.

En el ámbito de las Ciencias de la Actividad Física, estudios de los últimos años aportan grandes avances en algunas discapacidades; ejemplo de ello, es la discapacidad física que, por medio de un área de concentración de estudios en actividad motora adaptada, ha permitido desarrollarse en su clasificación, modalidad y normativa (Santana \& Duarte, 2009). Se ha evidenciado un progresivo interés de las personas con discapacidad física usuarios de silla de ruedas, por la práctica deportiva, prueba de ello, es que ha aumentado el número de participantes en distintos eventos para-deportivos y muy especialmente en Juegos Paralímpicos y Parapanamericanos. De la misma manera, se evidencia que el deporte adaptado con proyección paralímpica, se ha ido democratizando en las organiza- ciones escolares y, en los dos últimos años, en la educación superior universitaria. Por lo mismo, es necesario mencionar que, en la medida en que gran cantidad de jóvenes practiquen el deporte adaptado, éste se nutrirá y desarrollará el deporte Paralímpico; ya que el deporte y la actividad física son una fuente extraordinaria de placer físico, es la ocasión de expandir su personalidad (Parlebas citado en Cumellas \& Estrany, 2006).

El deporte Paralímpico, a su vez, además de considerar los factores sociales, psicológicos y físicos, exige un indiscutible nivel de desempeño deportivo por parte de los atletas que integran el movimiento. En este contexto, Cardozo, Cuervo y Murcia (2016) señalan que, en relación al rendimiento deportivo, será importante "poseer una composición corporal equilibrada y saludable que puede ser estudiada, a través de diversos métodos como el antropométrico de pliegues cutáneos de Durnin-Womersley u otro método de pliegues diseñado para tal fin" (p.69). En el ámbito de la antropometría, su valoración se convierte en un proceso necesario como una medida de diagnóstico de la salud (Cardozo, Cuervo y Murcia, 2016). En este sentido, es fundamental el seguimiento del atleta en situación de discapacidad durante todo el proceso de entrenamiento, ya que el rendimiento deportivo también se ve afectado por factores como la nutrición (Olivos, Cuevas, Álvarez y Jorquera, 2012), "la composición corporal, entre 
Pleticosic, Y., Luarte, C., Castelli, L., Barra, C., \& Pavez, V. (2019). Parámetros antropométricos de atletas paralímpicos usuarios de silla de ruedas de la región del Biobío. Revista Ciencias de la Actividad Física UCM, N²0(2), julio-diciembre, 1-14.

DOI: http://doi.org/10.29035/rcaf.20.2.4

otros factores, ha demostrado su utilidad tanto en la detección de talentos como en el control del rendimiento y la recuperación de lesiones (Chena, et al., 2015). Un mejor rendimiento deportivo, no solo dependerá de si las condiciones de entrenamiento físico, tanto desde el punto de vista cualitativo como cuantitativo son iguales, sino que, además, será en aquellos deportistas con condiciones morfológicas más favorables para la práctica del deporte en cuestión (Pradas de la Fuente, Carrasco, Martínez y Herrero 2007; Rodríguez, Castillo, Tejo y Rozowski, 2014). Según Hernández (2012 citado en Vesga, 2014) "el diseño de un perfil físico contribuye en la conformación de una base de datos que permite la comparación entre los más destacados, (...). Los parámetros antropométricos y fisiológicos forman parte del seguimiento integral de los talentosos" (p.7).

En cuanto a la obesidad, se conoce que es una enfermedad crónica de alta prevalencia en Chile, que también afecta a los atletas y paratletas, y se caracteriza por un mayor contenido de grasa corporal. En adultos, la obesidad se clasifica de acuerdo al Índice de Masa Corporal (IMC), por la buena correlación que presenta este indicador con la grasa corporal y riesgo para la salud a nivel poblacional (Moreno, 2012). Según la Organización Mundial de la Salud (2018), el IMC, es un instrumento válido para determinar el estado nutricional de la población. El riesgo de sobrepeso y obesi- dad es, habitualmente, mucho mayor en los niños, adolescentes y adultos con discapacidad, que en la población general (Ojeda y Cresp, 2011). Las personas con discapacidad tienen una mayor tendencia a la obesidad debido a factores como inactividad física y los malos hábitos alimentarios. Un estado nutricional controlado está estrechamente relacionado con la salud, puesto que los alimentos vienen a ser los responsables de mantener el cuerpo en la mejor disponibilidad para la ejecución desde un simple movimiento, así como también una gran cadena de patrones motrices (Ozols, 2007).

La presente investigación tiene como objetivo determinar el índice de masa corporal y porcentaje de grasa corporal de los atletas con proyección paralímpica, usuarios de silla de ruedas, pertenecientes a la región del Biobío, que servirán de orientación en los planes de entrenamiento respectivos.

\section{METODOLOGÍA}

El estudio es de enfoque cuantitativo, recolectando los datos a través de instrumentos estandarizados. El diseño es de tipo no experimental-transversal de alcance descriptivo, es decir, no hay manipulación de variables, se observan en un momento y se describen características de la muestra. La muestra es intencionada no probabilística: la elección no depende de la probabilidad, sino de las caracterís- 
ticas de la investigación. La muestra constó de 34 adultos 27 hombres y 7 mujeres entre 18 y 56 años. De las modalidades deportivas practicadas, 3 atletas fueron de la modalidad Paracanotaje, 26 atletas de básquetbol en silla de ruedas y 5 de Paratletismo. La evaluación antropométrica se realizó en el laboratorio de fisiología de la Universidad San Sebastián, a temperatura confortable, en las primeras horas de la mañana, previo a cualquier actividad física. Se determinó peso y estatura utilizando una balanza con tallímetro (Seca 220); el procedimiento utilizado para pesar a los deportistas que no podían sostenerse en bipedestación, fue sentarlos sobre un banco de madera previamente dispuesto sobre la báscula, y su valor independiente fue restado del valor final. La evaluación de la talla para los atletas que no pudieron sostenerse en bipedestación fue estimada mediante el protocolo de longitud acostado decúbito supino con adaptación del tallímetro. Se utilizó el protocolo según los requerimientos de la International Society for the Advancements of Kinanthopometry (ISAK, 2001) para medir los 6 pliegues cutáneos con un plicómetro Harpenden $(0,2 \mathrm{~mm})$. El porcentaje de grasa corporal se determinó, a través de la ecuación de Durnin \& Womersley (1974) donde se establece la densidad corporal considerando la sumatoria de 4 pliegues corporales: bíceps, tríceps, subescapular y suprailíaco. El índice de masa corporal (IMC), se calcu- ló según los protocolos indicados por la Organización Mundial de la Salud, a través de la fórmula de $\mathrm{P} / \mathrm{T}^{2}$, clasificando a los individuos en bajo peso, normal, sobrepeso y obeso (Ministerio de Salud [MINSAL], 2004).

Para caracterizar la muestra, se utilizó estadística descriptiva con estadígrafos de tendencia central; los datos fueran tabulados a través del paquete Microsoft Excel 2007® y analizados por medio del software GraphPad Prism versión 7.0 para MACOs®.

\section{RESULTADOS}

En el grupo de atletas de tres modalidades paradeportivas, usuarios de silla de ruedas, existe una gran variedad en la edad de los participantes (18 a 56 años), en la talla (1,23 a 1,79 m), en el peso (52 a 102 kg.), en el IMC (18 a 53) y en el \%CC (13,2 a 41,3). En la Tabla 1 se presentan los valores en relación al porcentaje de grasa corporal y evaluación nutricional, distribuidos en porcentajes medios y desviación 
Pleticosic, Y., Luarte, C., Castelli, L., Barra, C., \& Pavez, V. (2019). Parámetros antropométricos de atletas paralímpicos usuarios de silla de ruedas de la región del Biobío. Revista Ciencias de la Actividad Física UCM, N²0(2), julio-diciembre, 1-14.

DOI: http://doi.org/10.29035/rcaf.20.2.4

Tabla 1

Descripción antropométricas de atletas usuarios de silla de rueda, según sexo.

\begin{tabular}{|c|c|c|c|}
\hline & $\begin{array}{c}\text { General }(n=34) \\
(p \pm s d)\end{array}$ & $\begin{array}{c}\text { Masculino }(n=27) \\
(p \pm s d)\end{array}$ & $\begin{array}{c}\text { Femenino }(n=7) \\
(p \pm s d)\end{array}$ \\
\hline Edad & $35,4 \pm 11,2$ & $34,0 \pm 11,3$ & $41,0 \pm 9,63$ \\
\hline Estatura & $1,61 \pm 0,1$ & $1,63 \pm 3,1$ & $1,55 \pm 0,1$ \\
\hline Peso & $75,8 \pm 12,6$ & $77,6 \pm 13,0$ & $68,6 \pm 7,6$ \\
\hline IMC & $30,0 \pm 7,1$ & $30,4 \pm 7,4$ & $29,0 \pm 5,3$ \\
\hline$\%$ GC & $26,9 \pm 7,1$ & $24,6 \pm 5,4$ & $35,9 \pm 5,4$ \\
\hline
\end{tabular}

El principal resultado del estudio es que los deportistas presentan elevado porcentaje de grasa corporal $(26,9 \pm 7,1)$ y un estado nutricional que tiende a la obesidad $(30,0 \pm 7,1)$. El estado nutricional de los deportistas se obtuvo a través del IMC, los que se presentan en porcentajes, mientras que su categorización se expresa en: bajo peso, normal, sobrepeso y obesidad (Valdés, Godoy y Herrera, 2014). Las mujeres presentan un índice promedio de: $29,0 \mathrm{~kg} / \mathrm{m}^{2}$ y los varones de $30,4 \mathrm{~kg} / \mathrm{m}^{2}$. Este índice, según la OMS, clasifica a las mujeres dentro de la categoría de sobrepeso o pre-obeso, y a los varones en obesidad grado I o moderado (Moreno, 2012).

Por otra parte, el porcentaje de grasa corporal se obtuvo a través del método ISAK, la densidad, a través de la ecuación de Durnin y Womersley, para obtener el \% de grasa corporal con Siri. Las mujeres tienen un pro- medio de 34\%GC y los varones un 26,1 $\%$ CC.

La Tabla 2, indica las características antropométricas de los atletas usuarios de silla de ruedas, según modalidad deportiva. En la modalidad de Paracanotaje se evaluaron 3 deportistas, quienes presentaron la menor edad de la muestra con un promedio de 29 años, con un IMC y \%GC de $24 \mathrm{~kg} / \mathrm{m}^{2}$ y $24,3 \%$ respectivamente, lo que se clasifica con estado nutricional aceptable, pero con un porcentaje de grasa alto. Los deportistas de esta disciplina son los que presentan un mejor estado nutricional y menor porcentaje de grasa en comparación con el resto de los participantes. En Paratletismo, se evaluaron 5 atletas, cuyo promedio de edad fue 29,6 años. EI IMC arrojó un valor promedio de 27 $\mathrm{kg} / \mathrm{m}^{2}$, lo que se clasifica como Sobrepeso y presentan un \%GC de 26,76. La modalidad de Básquetbol en silla de 
ruedas presentó un total de 26 deportistas evaluados; la edad promedio es de 37,5 años, quienes poseen un IMC promedio de $31 \mathrm{~kg} / \mathrm{m}^{2}$, los que, según la clasificación de la OMS, se encuentran en Obesidad grado 1 y un \%GC de 27,29, lo que también indica Obesidad.

Tabla 2

Características antropométricas de los atletas usuarios de silla de ruedas, según modalidad deportiva.

\begin{tabular}{|c|c|c|c|}
\hline & $\begin{array}{c}\text { Básquetbol en silla } \\
\text { de ruedas }(n=26) \\
(p \pm s d)\end{array}$ & $\begin{array}{c}\text { Paracanotaje }(n=3) \\
(p \pm s d)\end{array}$ & 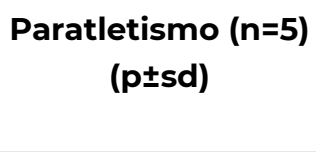 \\
\hline Edad (años) & $37,48 \pm 11,83$ & $29 \pm 4,36$ & $29,6 \pm 8,99$ \\
\hline Peso (kg) & $77,92 \pm 12,78$ & $66,43 \pm 9,46$ & $67,66 \pm 8,99$ \\
\hline Estatura (cm) & $1,61 \pm 0,13$ & $1,67 \pm 0,07$ & $1,61 \pm 0,14$ \\
\hline IMC & $31 \pm 7,18$ & $24 \pm 3,39$ & $27 \pm 7,21$ \\
\hline$\%$ CC (\%) & $27,29 \pm 4,79$ & $24,26, \pm 73,25$ & $26,76 \pm 13,33$ \\
\hline
\end{tabular}

\section{DISCUSIÓN}

El propósito de este estudio fue describir el estado nutricional y el porcentaje de grasa corporal de los deportistas en silla de ruedas de la región del Biobío, de tal forma, aportar a la escasa información sobre atletas chilenos usuarios de silla de ruedas. Los resultados muestran que el estado nutricional de los deportistas alcanza un promedio de 30,0 $\pm 7,1$, siendo para mujeres de 29,0 $\pm 5,3$ y en

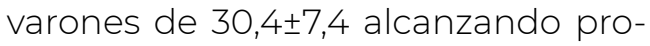
medios más altos, en referencia con otras poblaciones de deportistas chilenos paralímpicos. En el estudio de Durán-Agüero, Valdés-Badilla, Varas-Standen, Arrollo-Jofré \& Herrera-
Valenzuela (2016) el IMC alcanzó un promedio de $27,4 \mathrm{~kg} / \mathrm{m}^{2}$, lo que se encuentra por debajo de los resultados de este estudio. Esto podría explicarse por la inclusión de disciplinas que no usan sillas de ruedas. En un estudio de Rodríguez, Castillo, Tejo y Rozowski (2014) realizado a deportistas chilenos de alto rendimiento, donde se evaluó antropométricamente a 309 deportistas de 40 disciplinas, se encontró que su estado nutricional se presenta en categoría normal para una edad de 21 años, ambos en valores promedios; si esto se contrasta con los resultados del grupo de atletas usuarios de silla de rueda, nos encon- 
Pleticosic, Y., Luarte, C., Castelli, L., Barra, C., \& Pavez, V. (2019). Parámetros antropométricos de atletas paralímpicos usuarios de silla de ruedas de la región del Biobío. Revista Ciencias de la Actividad Física UCM, N²0(2), julio-diciembre, 1-14.

DOI: http://doi.org/10.29035/rcaf.20.2.4

tramos con un estado nutricional en categoría de obesidad para una edad promedio de 35,4 años. Esto puede explicarse por Ordonez et al. (2014) en el estudio con atletas de básquetbol en silla de ruedas con lesión medular crónica, donde se indica que el exceso de masa grasa es preocupante en los atletas usuarios de silla de ruedas. Este perfil se considera desventajoso para fines competitivos, ya que los altos índices de masa corporal deberán actuar como una sobrecarga que perjudicaría el desempeño en la ejecución de actitudes rápidas, tales como, cuando el cuerpo es proyectado horizontal y/o verticalmente las carreras y saltos (Gorla, et al., 2017; Piucco y Santos, 2009). En el estudio de Ojeda y Cresp (2011) se aprecia que el riesgo de obesidad en la población con discapacidad es mayor y para Monteverde (2015) el sobrepeso y la obesidad tendrían un efecto neto positivo sobre la presencia de discapacidades, sobre todo en edades comprendidas entre los 18 y 64 años.

En relación a los resultados de porcentaje de grasa corporal, estos alcanzan un promedio de $24,6 \pm 5,4$ para varones y $35,9 \pm 5,4$ para mujeres, siendo similares a los deportistas paralímpicos de élite chilenos, en el caso de los varones $(25,7 \%)$, pero es más alto en el caso de las mujeres (29,8\%) (Durán-Agüero, Valdés-Badilla, VarasStandend, Arroyo-Jofre \& Herrera-Valenzuela, 2016).
Respecto de los resultados obtenidos según la modalidad practicada por los atletas, encontramos que, el IMC promedio fue de $24 \pm$ y el \%GC de 24,26 \pm para los deportistas que practican paracanotaje. En un estudio de revisión bibliográfica, efectuado por Vesga (2014) se establece que, en los atletas convencionales, el porcentaje de grasa para kayak femenino osciló entre 22,4 \pm 5,83 \%, el masculino 6,9 \pm $0,79 \%$, y para los canoistas en un 7,2 $\pm 0,80 \%$, valores evidentemente inferiores al de los paracanoistas usuarios de silla de ruedas. Cabe destacar que, los resultados se compararon con atletas convencionales, ya que no existen evidencias de estudios nacionales realizados en atletas paralímpicos en esta modalidad.

En cuanto a los deportistas de la modalidad de paratletismo, el IMC promedio fue de 27t, y el \%GC de $26,76 \%$. Si lo comparamos con los resultados obtenidos por De Moura, Sampaio, Deldoto, Marques \& Bennemann (2013) en una caracterización nutricional de paratletas de Paraná, se obtuvo que la mayoría de los sujetos evaluados tenían sobrepeso (83.33\%) y el 16,7\% podía clasificarse como grado de obesidad I, lo que se asemeja con nuestra realidad nacional.

Los deportistas de básquetbol en silla de ruedas obtuvieron un IMC promedio de $31 \pm$ y \%GC de 27,29\%. En el estudio de Keil, Totosy De Zepetnek, Brooke-Wavell y Goosey-Tolfrey 
(2016) acerca de la medición de variables de composición corporal en doce basquetbolistas de élite masculinos en silla de ruedas, a través de absorciometría por rayos X (DXA), obtuvo como resultado un porcentaje medio de grasa corporal de $26.6 \%$ $\pm 6.5 \%$ (rango $16-33 \%$ ), lo que indica sobrepeso. Para Okorodudu et al., (2010) utilizando DXA, el nivel determinante para la obesidad en relación al porcentaje de grasa corporal es de $30 \%$ para las mujeres y el 25\% para los hombres. Cavedon, Zancanaro \& Milanese (2018), estudiaron la antropometría, composición corporal, y el rendimiento en 13 jugadoras de baloncesto en silla de ruedas y, a su vez, se compararon los resultados con un grupo de 23 hombres; el resultado en el porcentaje estimado de grasa corporal fue mayor en las mujeres (30.7 \pm $6.0 \%$ comparado con un grupo de 23 hombres $23.2 \pm 5.4 \%$ y llegaron a las siguientes conclusiones: la mayoría de las jugadoras de básquetbol en silla de ruedas tienen sobrepeso. Los hallazgos sugieren que las mujeres atléticas con discapacidad tienden a acumular exceso de grasa corporal. En Soares y De Sá Pereira (2002) los basquetbolistas (12) en silla de ruedas mostraron un predominio de porcentaje de grasa en la parte central del cuerpo (59,7\%), en relación a la suma de pliegues, seis de los atletas presentaron índices superiores a la media del grupo ( 26\%). Seis de los atletas presentaron índices superiores en el IMC en relación a la media del grupo
( 9\%). Cinco de ellos son los mismos atletas que presentan ambos indicadores por encima del promedio.

Es importante mencionar que, en la interpretación de los datos de este estudio, se debe tener en cuenta que la estimación de \%GC fue con una ecuación antropométrica desarrollada en sujetos sin discapacidad (Durnin \& Womersley, 1974).

\section{CONCLUSIÓN}

Este estudio proporcionó nueva información sobre las características antropométricas de los atletas con proyección paralímpica usuarios de silla de ruedas de la región del Biobío. Cabe destacar que existen diferencias en los resultados de los participantes relacionados con la edad, el sexo y la modalidad deportiva que practican.

Los resultados arrojados por la evaluación de los atletas paralímpicos indican que presentan un alto porcentaje de grasa corporal y clasificaciones de IMC que los categorizan en personas que presentan obesidad. Se sugiere que los atletas de proyección paralímpica sean acompañados con un plan de nutrición adecuada a los requerimientos energéticos de cada modalidad paradeportiva, teniendo en cuenta su clasificación funcional. De igual forma, trabajar en la pérdida del porcentaje de grasa corporal para un mejor desempeño, beneficiando su salud general. 
Pleticosic, Y., Luarte, C., Castelli, L., Barra, C., \& Pavez, V. (2019). Parámetros antropométricos de atletas paralímpicos usuarios de silla de ruedas de la región del Biobío. Revista Ciencias de la Actividad Física UCM, N²0(2), julio-diciembre, 1-14.

Proyectando este estudio, se sugiere, en investigaciones futuras, la necesidad de utilizar formas de evaluación antropométrica que contemplen otras discapacidades y modalidades paradeportivas, $y$, de igual forma, establecer correlaciones con test de desempeño físico-motores.

\section{REFERENCIAS BIBLIOGRÁFICAS}

Cardozo, L., Cuervo, Y., \& Murcia, J. (2016). Porcentaje de grasa corporal y prevalencia de sobrepeso - obesidad en estudiantes universitarios de rendimiento deportivo de Bogotá, Colombia. Revista Nutrición Clínica y Dietética Hospitalaria, 36(3), 68-75. Recuperado de https://revista. nutricion.org/PDF/cardozo.pdf

Cavedon, V., Zancanaro, C., \& Milanese, C. (2018). Anthropometry, Body Composition and Performance in Sport-Specific Field Test in Female Wheelchair Basketball Players. Frontiers in Physiolo9y, 568(9), 1-13. Recuperado de https://www.ncbi.nlm.nih.gov/ pubmed/29899703

Chena, M., Pérez-López, A., Álvarez, I., Bores, A., Ramos-Campo, D., Rubio-Arias, J., \& Valadés, D. (2015). Influencia de la composición corporal sobre el rendimiento en salto vertical dependiendo de la categoría de la formación y la demarcación en futbolistas. Nutrición Hospitalaria, 32(1), 299-307. Recuperado de http://scielo.isciii.es/ scielo.php?script=sci_arttext\&pi $d=$ S0212-16112015000700043

Cumellas, M., \& Estrany, C. (2006). Discapacidades motoras y sensoriales en primarias. Barcelona, España: INDE. 
De Moura, D., Sampaio, A., Deldoto, G., Marques, S. y Bennemann, R.M. (2013). Caracterização nutricional de indivíduos paratletas frequentadores de uma associação paradesportiva de maringá, Paraná. Simposio llevado a cabo en el VIII Encontro Interrnacional de Produção Científica Cesumar, Paraná Brasil. Recuperado de http://www. cesumar.br/prppge/pesquisa/ epcc2013/oit_mostra/Debora_ de_Moura.pdf

Durán-Agüero, S., Valdés-Badilla, P., Varas-Standend, C., Arroyo-Jofre, P., \& Herrera-Valenzuela $T$. (2016). Perfil Antropométrico de deportistas paralímpicos de élite chilenos. Revista Española de Nutrición Humana y Dietética, 20(4), 307:315. Recuperado de http://scielo.isciii.es/scieo.php?script=sci_arttext\&pid =S2174-51452016000400006

Durnin, J. \& Womersley, J. (1974). Body fat assessed from total body density and its estimation from skinfold thickness: Measurements on 481 men and women aged from 16 to 72 years. British Journal Nutrition, 32(1),7798. Recuperado de http://infocentre.jenage.de/assets/pdfs/ library/durnin_womersley_Br]Nutr_1974.pdf
Gorla, J., Costa, A., Castelli, L., Ferreira, C., Gavião, J., Duarte, E., \& Queiroga, M. (2017). Composic, ão corporal e perfil somatotípico de atletas da selec, ão brasileira de futebol de 5. Revista Brasileira de Ciências do Esporte, 39(1) 79-84. Recuperado de http://www.scielo.br/scielo.php?script=sci_arttext\&pid =S0101-32892017000100079

Hernández, J. (2000). El deporte para atender la diversidad: deporte adaptado y deporte inclusivo. Apuntes de Educación física y Deportes, 60(2), 46-53. Recuperado de https:// dialnet.unirioja.es/servlet/ articulo?codigo $=150327$

International Society for Advancements of Kinanthropometry. (2001). International standards for anthropometric assessment. Recuperado de https://docplayer.net/25335980-Internationalstandards-for-anthropometricassessment.html

Keil, M., Totosy De Zepetnek, J., Brooke-Wavell, K. \& GooseyTolfrey, V. (2016). Measurement precision of body composition variables in elite wheelchair athletes, using dual-energy $X$ ray absorptiometry. European Journal of Sport Science, 16(1), 65-71. Recuperado de https:// www.ncbi.nlm.nih.gov/pub$\mathrm{med} / 25307741$ 
Pleticosic, Y., Luarte, C., Castelli, L., Barra, C., \& Pavez, V. (2019). Parámetros antropométricos de atletas paralímpicos usuarios de silla de ruedas de la región del Biobío. Revista Ciencias de la Actividad Física UCM, N²0(2), julio-diciembre, 1-14.

DOI: http://doi.org/10.29035/rcaf.20.2.4

Ministerio de Salud (2004). Norma técnica de evaluación nutricional del niño de 6 a 18 años. Año 2003. Revista chilena de nutrición, 37(2), 128-137. Recuperado de https://dx.doi.org/10.4067/ s0717-75182004000200007

Monteverde, M. (2015). Exceso de peso y discapacidad en las personas mayores de la Argentina. Salud Colectiva, 77(4), 509-521. Recuperado de https://www.redalyc. org/pdf/731/73143274005.pdf

Moreno, M. (2012). Definición y clasificación de la Obesidad. Revista Médica Clínica Las Condes, 23(2), 124-128. Recuperado de https://www.clinicalascondes.cl/Dev_CLC/media/ Imagenes/PDF\%20revista\%20 m\%C3\%A9dica/2012/2\%20marzo/Dr_Moreno-4.pdf

Ojeda, R., \& Cresp, M. (2011). Correlación entre índice de masa corporal y circunferencia de cintura en una muestra de niños, adolescentes y adultos con discapacidad de Temuco, Chile. International Journal of Morphology, 29(4), 1326-1330. Recuperado de https://scielo.conicyt. cl/scielo.php?script=sci_arttext\& pid=S0717-95022011000400043
Okorodudu, D., Jumean, M, Montori, V., Romero-Corral, A., Somers, V., Erwin, P., et al. (2010). Diagnostic performance ofbodymassindex to identify obesity as defined by body adiposity: a systematic review and meta-analysis. International Journal of Obesity 34(5), 791-799. Recuperado de https://www.ncbi.nlm.nih.gov/ pubmed/20125098

Olivos, C., Cuevas A., Álvarez, V. \& Jorquera, C. (2012). Nutrición para el entrenamiento y la competición. Revista Médica Clínica Las Condes, 23(3) 253-261. Recuperado de https://www.clinicalascondes.cl/Dev_CLC/media/ Imagenes/PDF\%20revista\%20 m\% C3\%A9dica/2012/3\%20 mayo/6_Dra_Cuevas-8.pdf

Organización Mundial de la Salud (2018). Obesidad y sobrepeso. Recuperado de https:// www.who.int/es/news-room/ fact-sheets/detail/obesity-andoverweight.

Ordonez, F., Rosety, I., Fornieles, G., Rodriguez-Pareja, A., Rosety, M., Alvero-Cruz, J. \& RosetyRodriguez, M. (2014). Ecuación predictora de masa grasa corporal en deportistas con lesión medular crónica: estudio piloto. International Journal of Morphology, 32(1), 261-266. Recuperado de https://scielo.conicyt.cl/ scielo.php?script=sci_arttext\&pi $d=$ S0717-95022014000100043 
Ozols, M. (2007). Actividad Física y Discapacidad. Revista en Ciencias del Movimiento Humano y Salud, 4(2), 1-5. Recuperado de https://www.redalyc.org/ pdf/2370/237017534001.pdf

Pérez, J., Reina, R., Sanz, D. (2012). La Actividad Física Adaptada para personas con discapacidad en España: perspectivas científicas y de aplicación actual. Revista Cultura, Ciencia y Deporte, 7(21), 213-224. Recuperado de https://ccd.ucam.edu/index. php/revista/article/view/86

Piucco, T., Santos, S.G. (2009). Relacão entre percentual de gordura corporal, desempenho no salto vertical e impacto nos membros inferiores em atletas de voleibol. Fitness Performance Journal, 8(1), 9-15. Recuperado de https://www.redalyc.org/ pdf/751/75117016002.pdf

Pradas de la Fuente, F., Carrasco, L., Martínez, E. \& Herrero, R. (2007). Perfil antropométrico, somatotipo y composición corporal de jóvenes jugadores de tenis de mesa. Revista Internacional de Ciencias del Deporte, 3(7), 11-23. Recuperado de https://www.cafyd.com/REVISTA/ojs/index.php/ ricyde/article/view/48
Reina, R. (2010). La actividad física y deporte adaptado ante el Espacio Europeo de Enseñanza Superior. Sevilla, España: Wanceulen.

Rodríguez, X., Castillo, O., Tejo, C., \& Rozowski, J., (2014). Somatotipo de alto rendimiento de Santiago, Chile. Revista Chilena de Nutrición, 47(1) 29-39. Recuperado de https://scielo.conicyt.cl/ scielo.php?script=sci_arttext\&pi $d=$ S0717-75182014000100004

Santana, V., \& Duarte, E. (2009). Síndrome de Down: Informações, caminhos e histórias de amor. São Paulo, Brasil: Phorte.

Soares, S. \& De Sá Pereira, F. (2002). Avaliação antropométrica e de composição corporal de atletas paraolímpicos brasileiros. Revista Brasileira de Medicina do Esporte, 8(3), 84-91. Recuperado de http://www. scielo.br/scielo.php?pid=S1517922002000300004\&script=sci_ abstract\&tlng=pt

Torralba, M., Braz, M., \& Rubio, M. (2014). La motivación en el deporte adaptado. Journal Psychology, Society \& Education, 6(1), 27-40. Recuperado de http://ojs.ual. es/ojs/index.php/psye/article/ view/506 
Pleticosic, Y., Luarte, C., Castelli, L., Barra, C., \& Pavez, V. (2019). Parámetros antropométricos de atletas paralímpicos usuarios de silla de ruedas de la región del Biobío. Revista Ciencias de la Actividad Física UCM, N²0(2), julio-diciembre, 1-14.

DOI: http://doi.org/10.29035/rcaf.20.2.4

Valdés, P., Godoy, A., \& Herrera, T. (2014). Somatotipo, composición corporal, estado nutricional y condición física en personas con discapacidad visual que practican goalball. International Journal of Morphology, 32(1):183-189. Recuperado de https://scielo.conicyt.cl/scielo.php?script=sci_arttext\&pid =S0717-95022014000100031

Vesga, S. (2014). Perfil fisiológico y características antropométricas de los atletas de canotaje (canoa y kayak). Revisión. EFDeportes.com, Revista Digital, (196), 1. Recuperado de https:// www.efdeportes.com/efd796/ perfil-fisiologico-canotaje-canoa-y-kayak.htm

\section{Dirección para correspondencia}

Yazmina Virginia Pleticosic Ramírez

14 Magíster en Educación Física

Universidad San Sebastián, Concepción, Chile.

Número de registro en ORCID:

https://orcid.org/0000-0003-1110-6663

Contacto:

yazminapleticosic@gmail.com

Recibido: 29-07-2019

Aceptado: 30-09-2019 